Int. Journal of Math. Analysis, Vol. 8, 2014, no. 20, 995 - 1003

HIKARI Ltd, www.m-hikari.com

http://dx.doi.org/10.12988/ijma.2014.4392

\title{
New Iterative Algorithm for Variational Inequality Problem and Fixed Point Problem in Hilbert Spaces
}

\author{
Fuhai Wang \\ Department of Mathematics and Physics \\ North China Electric Power University \\ Baoding 071003, China \\ Minjiang Chen and Jianmin Song \\ School of Mathematics and Sciences \\ Shijiazhuang University of Economics \\ Shijiazhuang 050031, China
}

Copyright (c) 2014 Fuhai Wang, Minjiang Chen and Jianmin Song. This is an open access article distributed under the Creative Commons Attribution License, which permits unrestricted use, distribution, and reproduction in any medium, provided the original work is properly cited.

\begin{abstract}
In this paper, we introduce a new iterative scheme with a countable family of nonexpansive mappings for variational inequality problem in a Hilbert space and prove a strong convergence theorem for the iterative scheme.
\end{abstract}

Mathematics Subject Classification: 4705; 47H09; 47J25; 47N10

Keywords: Nonexpansive mappings; Hilbert spaces; Strong convergence; Variational inequality

\section{INTRODUCTION}

Let $H$ be a Hilbert space and $C$ be a nonempty closed convex subset of $H$. Let $F: H \rightarrow H$ be a nonlinear operator. The variational inequality problem on $F$ is to find a point $x^{*} \in C$ such that

$$
\left\langle F\left(x^{*}\right), x-x^{*}\right\rangle \geq 0, \quad \forall x \in C .
$$


The variational inequality problem is denoted by $V I(F, C)[5]$.

$F$ is called to be $\kappa$-Lischitzian and $\eta$-strongly monotone if there exist constants $\kappa, \eta>0$ such that

$$
\|F x-F y\| \leq \kappa\|x-y\|, \quad \text { for all } x, y \in H,
$$

and

$$
\langle F x-F y, x-y\rangle \geq \eta\|x-y\|^{2}, \quad \text { for all } x, y \in H,
$$

respectively.

It is well known that if $F$ is strongly monotone and Lipschitzian on $C$, then $V I(F, C)$ has a unique solution. An important problem is how to find a solution of $V I(F, C)$.

It is known that the $V I(F, C)$ is equivalent to the fixed point equation [16]

$$
u^{*}=P_{C}\left(u^{*}-\mu F\left(u^{*}\right)\right),
$$

where $P_{C}$ is the projection from $H$ onto $C$; i.e.,

$$
P_{C} x=\min _{y \in C}\|x-y\|, \quad \forall x \in H,
$$

and where $\mu>0$ is an arbitrarily fixed constant. So, the fixed point methods can be implemented to find a solution of the $V I(F, C)$.

The fixed point formulation (1.1) involves the projection $P_{C}$, which may not be easy to compute, due to the complexity of the convex set $C$. Hence, in order to reduce the complexity probably caused by the projection $P_{C}$, Yamada [16] introduced the hybrid steepest-descent method for solving the $V I(F, C)$ by replacing $P_{C}$ with a nonexpansive mapping $T$. Recall that a mapping $T: H \rightarrow H$ is called nonexpansive if for all $x, y \in H$, one holds

$$
\|T x-T y\| \leq\|x-y\| .
$$

The set of fixed points of $T$ is denoted by $F i x(T)$. More precisely, Yamada [16] gave the following iterative scheme:

$$
u_{0} \in H, \quad u_{n+1}=T u_{n}-\lambda_{n+1} \mu F\left(T u_{n}\right), \quad n \geq 0,
$$

where $T: H \rightarrow H$ is a nonexpansive mapping, $F$ is $\eta$-strongly monotone and $\kappa$-Lipschitzian on $C=F(T),\left\{\lambda_{n}\right\}$ is a sequence in $(0,1)$ and $\mu$ is a fixed number with $0<\mu<2 \eta / \kappa^{2}$. Yamada [16] proved that if the sequence $\left\{\lambda_{n}\right\}$ satisfies the conditions:

(L1) $\lim _{n \rightarrow \infty} \lambda_{n}=0$,

(L2) $\sum_{n=1}^{\infty} \lambda_{n}=\infty$,

(L3) $\lim _{n \rightarrow \infty}\left(\lambda_{n}-\lambda_{n+1}\right) / \lambda_{n+1}^{2}=0$,

then $\left\{u_{n}\right\}$ converges strongly to the unique solution of $V I(F, C)$.

Since Yamada's hybrid steepest-descent method for solving variational inequalities [16], there are much research on this aspect; see, e.g., [15, 19, 20, 21]. 
In 2011, Buong and Duong [2] introduced a new iterative algorithm, based on a combination of thy hybrid steepest-descent method for variational inequalities with the Krasnosel'skii-Mann type algorithm for fixed point problems. Very recently Zhou and Wang [23] simplified the algorithm of Buong and Duong and proved a strong convergence theorem for the variational inequality problem and fixed point problem on finite nonexpansive mappings in Hilbert space. In this paper, motivated by the work of Zhou and Wang [23], we introduce a new iterative algorithm for solving the solution of variational inequality problem and prove the solution is the common fixed point of a countable family of nonexpansive mappings in Hilbert space.

\section{Preliminaries}

Let $H$ be a Hilbert space. Let $T: H \rightarrow H$ be nonexpansive mapping and let $F: H \rightarrow H$ be a $\kappa$-Lipschitzian and $\eta$-strong monotone nonlinear operator.

Lemma 2.1 ([16]). Assume $\lambda \in(0,1)$ and $\mu \in\left(0,2 \eta / \kappa^{2}\right)$. Define a mapping $T^{\lambda}: H \rightarrow H$ by

$$
T^{\lambda} x=T x-\lambda \mu F(T x) \quad \forall x \in H .
$$

Then $\left\|T^{\lambda} x-T^{\lambda} y\right\| \leq(1-\lambda \tau)\|x-y\|$ for all $x, y \in H$, where $\tau=1-$ $\sqrt{1-\mu\left(2 \eta-\mu \kappa^{2}\right)} \in(0,1)$.

Obviously, if $T=I$, then $T^{\lambda}=I-\lambda \mu F$ and by Lemma 2.1 we have

$$
\left\|T^{\lambda} x-T^{\lambda} y\right\|=\|(I-\lambda \mu F) x-(I-\lambda \mu F) y\| \leq(1-\lambda \tau)\|x-y\|
$$

for all $x, y \in H$.

Lemma $2.2([7])$. Let $\left\{s_{n}\right\},\left\{c_{n}\right\}$ be the sequences of nonnegative real numbers and let $\left\{a_{n}\right\} \subset(0,1)$. Suppose $\left\{b_{n}\right\}$ is a real number sequence such that

$$
s_{n+1} \leq\left(1-a_{n}\right) s_{n}+b_{n}+c_{n}, \quad n \geq 0 .
$$

Assume $\sum_{n=0}^{\infty} c_{n}<\infty$. Then the following results hold:

(1) If $b_{n} \leq \beta a_{n}$ where $(\beta \geq 0)$, then $\left\{s_{n}\right\}$ is a bounded sequence.

(2) If we have

$$
\sum_{n=0}^{\infty} a_{n}=\infty \quad \text { and } \quad \limsup _{n \rightarrow \infty} \frac{b_{n}}{a_{n}} \leq 0,
$$

then $\lim _{n \rightarrow \infty} s_{n}=0$.

Let $T: H \rightarrow H$ be a nonexpansive mapping. Let $\alpha \in(0,1)$. Define the mapping $S=\alpha I+(1-\alpha) T$. Then the mapping $S$ is said to be an averaged mapping with $F i x(T)=F i x(S)$. Furthermore, on a family of averaged mappings, we have the following conclusion.

Lemma $2.3([1,12])$. Let $\left\{T_{i}\right\}_{i=1}^{N}$ be the averaged mappings on $H$ and assume that $\cap_{i=1}^{N} F i x\left(T_{i}\right) \neq \emptyset$. Then $\cap_{i=1}^{N} F i x\left(T_{i}\right)=F i x\left(T_{1} \ldots s T_{N}\right)$. 
Lemma 2.4 ([13]). Let $\left\{a_{n}\right\}$ be a sequence of nonnegative real numbers satisfying the following condition

$$
a_{n+1} \leq\left(1-b_{n}\right) a_{n}+b_{n} c_{n}
$$

where $\left\{b_{n}\right\}$ and $\left\{c_{n}\right\}$ are sequences of real numbers such that

$$
b_{n} \in[0,1], \sum_{n=0}^{\infty} b_{n}=\infty \text { and } \limsup _{n \rightarrow \infty} c_{n} \leq 0
$$

Then $\lim _{n \rightarrow \infty} a_{n}=0$.

\section{MAin RESUlts}

Theorem 3.1. Let $H$ be a real Hilbert space and $F: H \rightarrow H$ be an L-Lipschitz continuous and $\eta$-strongly monotone mapping. Let $\left\{T_{i}\right\}_{i=1}^{\infty}$ be a countable family of nonexpansive of $H$ such that $C=\cap_{i=1}^{\infty} F i x\left(T_{i}\right) \neq \emptyset$. Let $\left\{\lambda_{n}\right\}_{n=1}^{\infty}$, $\left\{\beta_{n}\right\}_{n=1}^{\infty},\left\{\alpha_{n}\right\}_{n=1}^{\infty}$ be sequences in $(0,1)$. Assume that $\left\{\beta_{n}\right\}$ is strictly decreasing and let $\beta_{0}=1$ and $\mu \in\left(0,2 \eta / L^{2}\right)$. Suppose that the following conditions hold:

$$
\lim _{n \rightarrow \infty} \lambda_{n}=0, \sum_{n=1}^{\infty} \lambda_{n}=\infty, \lim _{n \rightarrow \infty} \frac{\lambda_{n-1}}{\lambda_{n}}=1 \text { and } \sum_{n=1}^{\infty}\left|\beta_{n}-\beta_{n+1}\right|<\infty .
$$

Then the sequence $\left\{x_{n}\right\}$ generated by the following manner:

$$
x_{1} \in H, x_{n+1}=\left(I-\lambda_{n} \mu F\right)\left(\beta_{n} x_{n}+\sum_{i=1}^{n}\left(\beta_{i-1}-\beta_{i}\right) \Pi_{j=1}^{i} U_{j} x_{n}\right), \quad \forall n \geq 1
$$

where $U_{i}=\alpha_{i} I+\left(1-\alpha_{i}\right) T_{i}$, converges strongly to the unique solution $x^{*}$ of the variational inequality:

$$
\left\langle F\left(x^{*}\right), x-x^{*}\right\rangle \geq 0, \quad \forall x \in C .
$$


Proof.

$$
\begin{aligned}
\left\|x_{n+1}-p\right\| & =\left\|\left(I-\lambda_{n} \mu F\right)\left(\beta_{n} x_{n}+\sum_{i=1}^{n}\left(\beta_{i-1}-\beta_{i}\right) \Pi_{j=1}^{i} U_{i} x_{n}\right)-p\right\| \\
& =\left\|\left(I-\lambda_{n} \mu F\right)\left(\beta_{n} x_{n}+\sum_{i=1}^{n}\left(\beta_{i-1}-\beta_{i}\right) \Pi_{j=1}^{i} U_{i} x_{n}\right)-(I-\lambda \mu F) p+\lambda_{n} \mu F(p)\right\| \\
& \leq\left\|\left(I-\lambda_{n} \mu F\right)\left(\beta_{n} x_{n}+\sum_{i=1}^{n}\left(\beta_{i-1}-\beta_{i}\right) \Pi_{j=1}^{i} U_{i} x_{n}\right)-(I-\lambda \mu F) p\right\|+\lambda_{n} \mu\|F(p)\| \\
& \leq\left(1-\tau \lambda_{n}\right)\left\|\beta_{n} x_{n}+\sum_{i=1}^{n}\left(\beta_{i-1}-\beta_{i}\right) \Pi_{j=1}^{i} U_{i} x_{n}-p\right\|+\lambda_{n} \mu\|F(p)\| \\
& \leq\left(1-\tau \lambda_{n}\right)\left[\beta_{n}\left\|x_{n}-p\right\|+\left(1-\beta_{n}\right)\left\|x_{n}-p\right\|\right]+\lambda_{n} \mu\|F(p)\| \\
& =\left(1-\tau \lambda_{n}\right)\left\|x_{n}-p\right\|+\tau \lambda_{n} \frac{\mu}{\tau}\|F(p)\| \\
& \leq \max \left\{\left\|x_{0}-p\right\|, \frac{\mu}{\tau}\|F(p)\|\right\} .
\end{aligned}
$$

So, $\left\{x_{n}\right\}$ is bounded and so are $\left\{U_{i} x_{n}\right\}$ for each $i \geq 1$.

Next we prove that $x_{n+1}-x_{n} \rightarrow 0$ as $n \rightarrow \infty$. Let $y_{n}=\beta_{n} x_{n}+\sum_{i=1}^{n}\left(\beta_{i-1}-\right.$ $\left.\beta_{i}\right) W_{i} x_{n}$, where $W_{i}=\prod_{j=1}^{i} U_{j}$ for each $i \geq 1$. We rewrite (3.1) as follows:

$$
x_{n+1}=\left(I-\lambda_{n} \mu F\right) y_{n}, \quad \forall n \geq 1,
$$

Then

$$
\begin{aligned}
\left\|y_{n}-y_{n-1}\right\|= & \left\|\beta_{n} x_{n}+\sum_{i=1}^{n}\left(\beta_{i-1}-\beta_{i}\right) W_{i} x_{n}-\left(\beta_{n-1} x_{n-1}+\sum_{i=1}^{n-1}\left(\beta_{i-1}-\beta_{i}\right) W_{i} x_{n-1}\right)\right\| \\
= & \| \beta_{n}\left(x_{n}-x_{n-1}\right)+\left(\beta_{n}-\beta_{n-1}\right) x_{n-1}+\sum_{i=1}^{n}\left(\beta_{i-1}-\beta_{i}\right)\left(W_{i} x_{n}-W_{i} x_{n-1}\right) \\
& \quad+\left(\beta_{n-1}-\beta_{n}\right) W_{n} x_{n-1} \| \\
\leq & \beta_{n}\left\|x_{n}-x_{n-1}\right\|+\left|\beta_{n-1}-\beta_{n}\right|\left\|x_{n-1}\right\|+\sum_{i=1}^{n-1}\left(\beta_{i-1}-\beta_{i}\right)\left\|W_{i} x_{n}-W_{i} x_{n-1}\right\| \\
& \quad+\left|\beta_{n-1}-\beta_{n}\right||| W_{n} x_{n-1} \| \\
\leq & \beta_{n}\left\|x_{n}-x_{n-1}\right\|+\left|\beta_{n-1}-\beta_{n}\right|\left\|x_{n-1}\right\|+\sum_{i=1}^{n-1}\left(\beta_{i-1}-\beta_{i}\right)\left\|x_{n}-x_{n-1}\right\| \\
& \quad+\left|\beta_{n-1}-\beta_{n}\right||| W_{n} x_{n-1} \| \\
= & \beta_{n}\left\|x_{n}-x_{n-1}\right\|+\left|\beta_{n-1}-\beta_{n}\right|\left(\left\|x_{n-1}\right\|+\left\|W_{n} x_{n-1}\right\|\right)+\left(1-\beta_{n}\right)\left\|x_{n}-x_{n-1}\right\| \\
= & \left\|x_{n}-x_{n-1}\right\|+\left|\beta_{n-1}-\beta_{n}\right| M,
\end{aligned}
$$


where $M=\sup _{n \geq 1}\left\{\left\|x_{n-1}\right\|+\left\|W_{n} x_{n-1}\right\|\right\}$. Then

$$
\begin{aligned}
\left\|x_{n+1}-x_{n}\right\| & =\left\|\left(I-\lambda_{n} \mu F\right) y_{n}-\left(I-\lambda_{n-1} \mu F\right) y_{n-1}\right\| \\
& =\left\|\left(I-\lambda_{n} \mu F\right)\left(y_{n}-y_{n-1}\right)+\left(\left(I-\lambda_{n} \mu F\right)-\left(I-\lambda_{n-1} \mu F\right)\right) y_{n-1}\right\| \\
& =\left\|\left(I-\lambda_{n} \mu F\right)\left(y_{n}-y_{n-1}\right)+\left(\lambda_{n-1} \mu F-\lambda_{n} \mu F\right) y_{n-1}\right\| \\
& =\left\|\left(I-\lambda_{n} \mu F\right)\left(y_{n}-y_{n-1}\right)+\left(\lambda_{n-1}-\lambda_{n}\right) \mu F y_{n}\right\| \\
& \leq\left(1-\lambda_{n} \tau\right)\left\|y_{n}-y_{n-1}\right\|+\left|\lambda_{n-1}-\lambda_{n}\right| \mu\left\|F y_{n}\right\| \\
& \leq\left(1-\lambda_{n} \tau\right)\left(\left\|x_{n}-x_{n-1}\right\|+\left|\beta_{n-1}-\beta_{n}\right| M\right)+\left|\lambda_{n-1}-\lambda_{n}\right| \mu\left\|F y_{n}\right\| \\
& \leq\left(1-\lambda_{n} \tau\right)\left\|x_{n}-x_{n-1}\right\|+\left|\beta_{n-1}-\beta_{n}\right| M+\left|\lambda_{n-1}-\lambda_{n}\right| \mu M^{\prime},
\end{aligned}
$$

where $M^{\prime}=\sup _{n \geq 1}\left\{\left\|F y_{n}\right\|\right\}$. By Lemma 2.2 we conclude that

$$
\lim _{n \rightarrow \infty}\left\|x_{n+1}-x_{n}\right\|=0 .
$$

Since $\lambda_{n} \rightarrow 0$ as $n \rightarrow \infty$, it follows from (3.3) that

$$
\left\|x_{n+1}-y_{n}\right\| \rightarrow 0, \text { as } n \rightarrow \infty
$$

Furthermore, by (3.4) and (3.5) we get

$$
\lim _{n \rightarrow \infty}\left\|x_{n}-y_{n}\right\|=0
$$

Note that $y_{n}-x_{n}=\sum_{i=1}^{n}\left(\beta_{i-1}-\beta_{i}\right)\left(W_{i} x_{n}-x_{n}\right)$ and $\left\{\beta_{n}\right\}$ is a strictly decreasing sequence. So, for each $i \geq 1$, we have

$$
\left\|W_{i} x_{n}-x_{n}\right\| \leq \sum_{i=1}^{n}\left(\beta_{i-1}-\beta_{i}\right)\left\|W_{i} x_{n}-x_{n}\right\|=\left\|y_{n}-x_{n}\right\| \rightarrow 0, \text { as } n \rightarrow \infty \text {. }
$$

Next we prove that $\limsup _{n \rightarrow \infty}\left\langle-F\left(x^{*}\right), x_{n+1}-x^{*}\right\rangle \leq 0$. To prove this, we pick a subsequence $\left\{x_{n_{i}}\right\}$ of $\left\{x_{n}\right\}$ such that

$$
\limsup _{n \rightarrow \infty}\left\langle-F\left(x^{*}\right), x_{n}-x^{*}\right\rangle=\lim _{i \rightarrow \infty}\left\langle-F\left(x^{*}\right), x_{n_{i}}-x^{*}\right\rangle .
$$

Without loss of generality, we may further assume that $x_{n_{i}} \rightarrow \hat{x}$ for some $\hat{x} \in C$. By (3.7) and demiclosed principle we get $\hat{x} \in F i x\left(W_{i}\right)$ for each $i \geq 1$. Furthermore, by the definition of $W_{i}$ and Lemma 2.3 we conclude that $\hat{x} \in \cap_{j=1}^{\infty} F i x\left(T_{j}\right)$. Since $x^{*}$ is the unique solution of $(3.2)$, we obtain

$$
\limsup _{n \rightarrow \infty}\left\langle F\left(x^{*}\right), x^{*}-x_{n}\right\rangle=\lim _{i \rightarrow \infty}\left\langle F\left(x^{*}\right), x^{*}-\hat{x}\right\rangle \leq 0 .
$$

Finally, we prove that $x_{n} \rightarrow x^{*}$ as $n \rightarrow \infty$. 
By virtue of (3.3) and Lemma 2.1, we have

$$
\begin{aligned}
& \left\|x_{n+1}-x^{*}\right\|^{2} \\
= & \left\|\left(I-\lambda_{n} \mu F\right) y_{n}-x^{*}\right\|^{2} \\
= & \left\|\left(I-\lambda_{n} \mu F\right) y_{n}-\left(I-\lambda_{n} \mu F\right) x^{*}-\lambda_{n} \mu F\left(x^{*}\right)\right\|^{2} \\
= & \left\|\left(I-\lambda_{n} \mu F\right) y_{n}-\left(I-\lambda_{n} \mu F\right) x^{*}\right\|^{2}+2 \lambda_{n} \mu\left\langle F\left(x^{*}\right),\left(I-\lambda_{n} \mu F\right) x^{*}-\left(I-\lambda_{n} \mu F\right) y_{n}\right\rangle \\
& +\lambda_{n}^{2} \mu^{2}\left\|F\left(x^{*}\right)\right\|^{2} \\
\leq & \left(1-\tau \lambda_{n}\right)\left\|x_{n}-x^{*}\right\|^{2}+2 \lambda_{n} \mu\left\langle F\left(x^{*}\right), x^{*}-x_{n}\right\rangle+2 \lambda_{n} \mu\left\|F\left(x^{*}\right)\right\|\left\|x_{n}-y_{n}\right\| \\
& +2 \lambda_{n}^{2} \mu^{2}\left(\left\|F\left(x^{*}\right)\right\|+\left\|F\left(y_{n}\right)\right\|\right)+\lambda_{n}^{2} \mu^{2}\left\|F\left(x^{*}\right)\right\|^{2} \\
= & \left(1-\tau \lambda_{n}\right)\left\|x_{n}-x^{*}\right\|^{2}+2 \lambda_{n} \mu\left\langle F\left(x^{*}\right), x^{*}-x_{n}\right\rangle+2 \lambda_{n} \mu\left\|F\left(x^{*}\right)\right\|\left\|x_{n}-y_{n}\right\| \\
& +\lambda_{n}^{2} \mu^{2}\left(2\left\|F\left(x^{*}\right)\right\|+2\left\|F\left(y_{n}\right)\right\|+\left\|F\left(x^{*}\right)\right\|^{2}\right) \\
\leq & \left(1-\tau \lambda_{n}\right)\left\|x_{n}-x^{*}\right\|^{2}+\lambda_{n} \tau c_{n},
\end{aligned}
$$

where

$$
c_{n}=\frac{\mu}{\tau}\left(2\left\langle F\left(x^{*}\right), x^{*}-x_{n}\right\rangle+2\left\|F\left(x^{*}\right)\right\|\left\|x_{n}-y_{n}\right\|+\lambda_{n} \mu L\right)
$$

and

$$
L=\sup _{n \geq 1}\left(2\left\|F\left(x^{*}\right)\right\|+2\left\|F\left(y_{n}\right)\right\|+\left\|F\left(x^{*}\right)\right\|^{2}\right) \text { is a fixed constant. }
$$

From (3.6)-(3.8), we know that $\lim _{\sup _{n \rightarrow \infty}} c_{n} \leq 0$. By Lemma 2.4, we conclude that $x_{n} \rightarrow x^{*}$ as $n \rightarrow \infty$. This completes the proof.

Corollary 3.1. Let $H$ be a real Hilbert space and $F: H \rightarrow H$ be an L-Lipschitz continuous and $\eta$-strongly monotone mapping. Let $\left\{T_{i}\right\}_{i=1}^{N}$ be a family of nonexpansive of $H$ such that $C=\cap_{i=1}^{N} F i x\left(T_{i}\right) \neq \emptyset$. Let $\left\{\lambda_{n}\right\}_{n=1}^{\infty},\left\{\beta_{n}\right\}_{n=1}^{\infty}$ and $\left\{\alpha_{n}\right\}_{n=1}^{\infty}$ be sequences in $(0,1)$. Assume that $\mu \in\left(0,2 \eta / L^{2}\right)$. Suppose that the following conditions hold:

$$
\lim _{n \rightarrow \infty} \lambda_{n}=0, \sum_{n=1}^{\infty} \lambda_{n}=\infty, \lim _{n \rightarrow \infty} \frac{\lambda_{n-1}}{\lambda_{n}}=1 \text { and } \sum_{n=1}^{\infty}\left|\beta_{n}-\beta_{n+1}\right|<\infty .
$$

Then the sequence $\left\{x_{n}\right\}$ generated by the following manner:

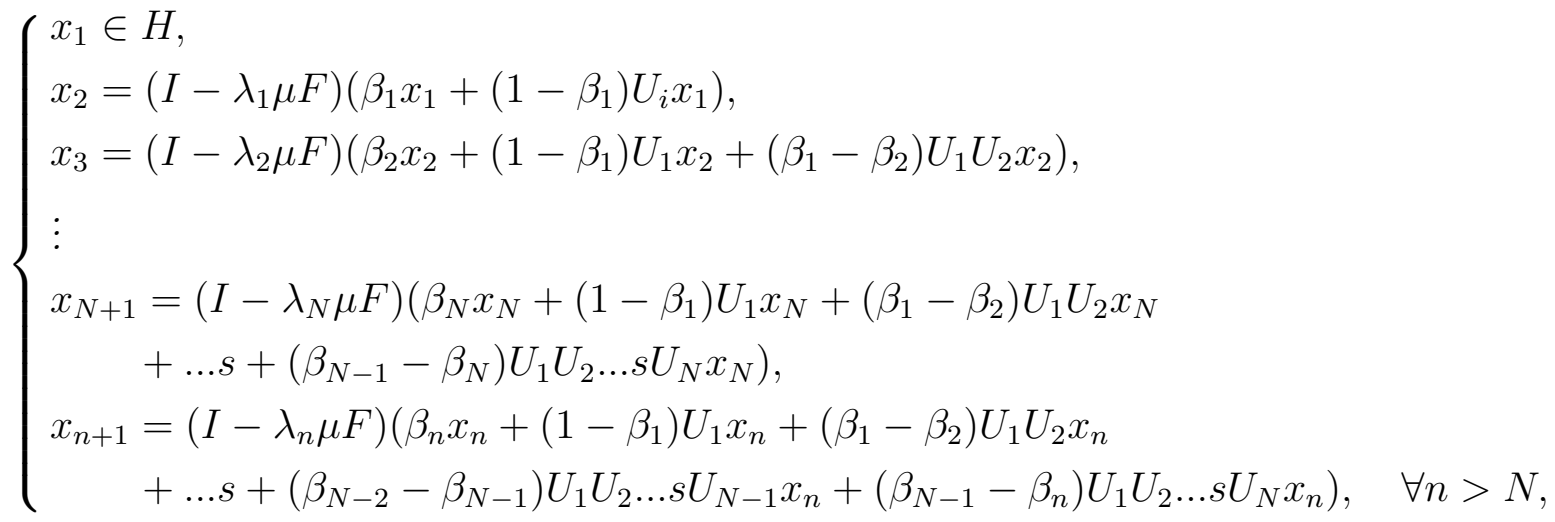


where $U_{i}=\alpha_{i} I+\left(1-\alpha_{i}\right) T_{i}(i=1,2, \ldots s, N)$, converges strongly to the unique solution $x^{*}$ of the variational inequality:

$$
\left\langle F\left(x^{*}\right), x-x^{*}\right\rangle \geq 0, \quad \forall x \in C .
$$

Corollary 3.2. Let $H$ be a real Hilbert space and $F: H \rightarrow H$ be an $L$ Lipschitz continuous and $\eta$-strongly monotone mapping. Let $T$ be a nonexpansive of $H$ such that $C=F i x(T) \neq \emptyset$. Let $\left\{\lambda_{n}\right\}_{n=1}^{\infty}$ and $\left\{\beta_{n}\right\}_{n=1}^{\infty}$ be sequences in $(0,1)$. Assume that $\mu \in\left(0,2 \eta / L^{2}\right)$. Suppose that the following conditions hold:

$$
\lim _{n \rightarrow \infty} \lambda_{n}=0, \sum_{n=1}^{\infty} \lambda_{n}=\infty, \lim _{n \rightarrow \infty} \frac{\lambda_{n-1}}{\lambda_{n}}=1 \text { and } \sum_{n=1}^{\infty}\left|\beta_{n}-\beta_{n+1}\right|<\infty .
$$

Then the sequence $\left\{x_{n}\right\}$ generated by the following manner:

$$
x_{1} \in H, x_{n+1}=\left(I-\lambda_{n} \mu F\right)\left(\beta_{n} x_{n}+\left(1-\beta_{n}\right) U x_{n}\right), \quad \forall n \geq 1,
$$

where $U=\alpha I+(1-\alpha) T$ and $0<\alpha<1$, converges strongly to the unique solution $x^{*}$ of the variational inequality:

$$
\left\langle F\left(x^{*}\right), x-x^{*}\right\rangle \geq 0, \quad \forall x \in C .
$$

\section{Acknowledgements}

This work is supported by the Scientific Research Fund of Hebei Provincial Education Department (Grant No. Z2013110), Multi-level Advanced Mathematics Research Fund (Grant No. GH 132068) and HeiBei Education Department (Grant No. 936101101).

\section{REFERENCES}

[1] C. Byrne, A unified treatment of some iterative algorithms in signal processing and image reconstruction. Inverse Probl. 20 (2004) 103-120.

[2] D. Buong, L.T. Duong, An explicit iterative algorithm for a class of variational inequalities in Hilbert spaces, J. Optim. Theory Appl. 151 (2011) 513-524.

[3] F. Cianciaruso, G. Marino, L. Muglia, Y. Yao, "On a two-steps algorithm for hierarchical fixed points problems and variational inequalities," Journal Inequality Application, 2009 (2009), doi:10.1155/2009/208692, 13 pages, Article ID 208692.

[4] K. Goebel, W.A. Kirk, Topics in Metric Fixed Point Theory, in: Cambridge Studies in Advanced Mathematics, vol. 28, Cambridge University Press, 1990.

[5] D. Kinderlehrer and G. Stampacchia, An introduction to Variational inequalities and their applications, Academic Press, New York, NY, 1980.

[6] P.E. Mainge, A. Moudafi, "Strong convergence ofan iterative method for hierarchical fixed point problems," Pacific Journal Optimization, vol. 3, pp. 529-538, 2007.

[7] P.E. Maingé, "Approximation methods for common fixed points of nonexpansive mappings in Hilbert spaces," Journal Mathematics Analysis and Application, vol. 325, pp. 469-479, 2007.

[8] G. Marino, H.K. Xu, "Explicit hierarchical fixed point approach to variational inequalities," Journal of Optimization Theory and Applications, vol. 149, pp. 61-78, 2011.

[9] G. Marino, H.K. Xu, "A general iterative method for nonexpansive mappings in Hilbert spaces," Journal Mathematics Analysis and Application, vol. 318, pp. 43-52, 2006. 
[10] A. Moudafi, "Krasnoselski-Mann iteration for hierarchical fixed-point problems," Inverse Problems, vol. 23, pp. 1635-1640, 2007.

[11] A. Moudafi, "Viscosity approximation methods for fixed-points problems," Journal of Mathematical Analysis and Applications, vol. 241, pp. 46-55, 2000.

[12] H.K. Xu, Averaged mappings and the gradient-projection algorithm, J. Optim. Theory Appl. 150 (2011) 360-378.

[13] H.K. Xu, An iterative aporoach to quadratic optimization, J. Optim. Theory Appl. 116 (2003) 659-678.

[14] H.-K. Xu, "Viscosity approximation methods for nonexpansive mappings," Journal of Mathematical Analysis and Applications, vol. 298, pp. 279-291, 2004.

[15] H.K. Xu and T.H. Kim, Convergence of hybrid steepest-descent methods for variational inequalities, J. Optim. Theory Appl. 119 (2003) 185-201.

[16] I. Yamada, The hybrid steepest descent method for the variational inequality problem over the intersection of fixed point sets of nonexpansive mappings, in: D. Butnarium Y. Censor, S. Reich (Eds.), Inherently Parallel Algorithm for Feasibility and Optimization, Elsevier, 2001, 473-504.

[17] I. Yamada and N. Ogura, Hybrid steepest descent method for the variational inequality problem over the fixed point set of certain quasi-nonexpansive mappings, Numer. Funct. Anal. Optim. 25 (2004) 619-655.

[18] Y.H. Yao, Y.J. Cho, Y.C. Liou, "Iterative algorithms for hierarchical fixed points problems and variational inequalities," Mathematical and Computer Modelling, vol. 52, pp. 1697-1705, 2010.

[19] L.C. Zeng, Q.H. Ansari and S.Y. Wu, Strong convergence theorems of relaxed hybrid steepest-descent methods for variational inequalities, Taiwanese J. Math. 10 (2006) $13-29$.

[20] L.C. Zeng, N.C. Wong and J.C. Yao, Convergence analysis of modified hybrid steepestdescent methods with variable parameters for variational inequalities, J. Optim. Theory Appl. 132 (2007) 51-69.

[21] L.C. Ceng, H.K. Xu and J.C. Yao, A hybrid steepest-descent method for variational inequalities in Hilbert spaces, Applicable Analysis 87 (2008) 575-589.

[22] H. Y. Zhou, convergence theorems of fixed points for $\kappa$-strict pseudo-contractions in Hilbert spaces, Nonlinear Anal. 69, (2008) 456-462.

[23] H.Y. Zhuo, P.Y. Wang, A simpler explicit iterative algorithm for a class of variational inequalities in Hilbert spaces, J. Optim. Theory Appl. Doi 10. 1007/s10957-013-0470-x.

\section{Received: March 1, 2014}

